\title{
Erratum to: The Proper Treatment of Linguistic Ambiguity in Ordinary Algebra
}

Christian Wurm and Timm Lichte

\section{Erratum to: \\ Chapter "The Proper Treatment of Linguistic Ambiguity \\ in Ordinary Algebra" in: A. Foret et al. (Eds.): \\ Formal Grammar, LNCS 9804, https://doi.org/10.1007/978-3-662-53042-9_18}

This is a correction note to the paper starting on p. 306. There is an error in the proof of Lemma 1, which states that the axiom

At least one of $a \leq a \| b$ or $b \leq a \| b$ holds

together with the other axioms entails the stronger statement that either $a=a \| b$ or $b=a \| b$. The proof of this lemma is incorrect, and the claim is wrong: we can construct an algebra with 4 elements $\{0,1,0\|1,1\| 0\}$ with the obvious Boolean algebra order, and $\|$ defined by the margin property: $a\|b\| c=a \| c$, with $a, b \in$ $\{0,1\}, c$ an arbitrary term. It is not difficult to check that this is an ambiguous algebra in the sense of Section 4 of the paper, yet $0 \neq 0 \| 1 \neq 1$. As almost all later results are based upon Lemma 1, they are technically unproved. However, all problems can be remedied very easily by changing $(\| 3)$ from the paper to:

$\left(\| 3^{\prime}\right) \quad$ At least one of $a=a \| b$ or $b=a \| b$ holds

Hence to make the paper correct, all we need is a slightly different axiom $\left(\| 3^{\prime}\right)$, and Lemma 1 becomes basically part of the definition, so all problems are solved. So far our correction; there are two notes which might be interesting to the reader:

The original online version of this chapter can be found at https://doi.org/10.1007/978-3-662-53042-9_18 
Note 1 From the point of view of the linguistic motivation of the axioms, $\left(\| 3^{\prime}\right)$ is actually more natural than the original $(\| 3)$, because it basically states that an ambiguous meaning is supposed to intend one of the meanings between which it is ambiguous. The weaker (\|3) just states that it is supposed to entail one of these meanings, which is not what we would intuitively think. Actually, the authors of the paper preferred $(\| 3)$ over $\left(\| 3^{\prime}\right)$ not on a conceptual base, but rather because it is simply weaker and they believed the two to be equivalent anyway (this is what Lemma 1 of the mentioned paper states).

Note 2 In (Wurm, 2017), the authors have introduced the class of universal distribution algebras (UDA), which is still weaker. If we take the class of ambiguous algebras as introduced in the 2016 paper and add an axiom for $\|$-associativity $(a\|(b \| c)=(a \| b)\| c$, which does not seem derivable so far), then it is not difficult to show that UDA subsumes this class. What is interesting is that UDA seems to have the same equational theory as ambiguous algebras in the strong sense (with $\left(\| 3^{\prime}\right)$ ). Now since the class as defined in 2016, with associativity added, lies in between the two, it is neatly characterized by this (yet unpublished) result.

\section{Reference}

Wurm, C.: The logic of ambiguity: the propositional case. In: Foret, A., Muskens, R., Pogodalla, S. (eds.) Formal Grammar. 22th Conference, FG 2017, Toulouse, France, July 2017, Proceedings. Lecture Notes in Computer Science, vol. 10686. Springer (2017) 